Tohoku J. Exp. Med., 1990, 161, 101-110

\title{
Dose Dependency of Intratracheal Elastase-Induced Changes in Pressure Volume Curve and Morphometry in Rat Lungs
}

\author{
Shinobu Sato, Shuichi Kato, Hihumi Takahashi, \\ Yasusi Arisaka and Keiju Takahashi \\ The First Department of Internal Medicine, Yamagata \\ University School of Medicine, Yamagata 990-23
}

\begin{abstract}
Sato, S., Kato, S., Takahashi, H., Arisaka, Y. and Takahashi, K. Dose Dependency of Intratracheal Elastase-Induced Changes in Pressure Volume Curve and Morphometry in Rat Lungs. Tohoku J. Exp. Med., 1990, 161 (2), 101-110 - We studied the dose-dependency of the lung pressure-volume curve $(n=37)$ and morphometry $(n=30)$ with intratracheally administered elastase. Four weeks after elastase was instilled at dosages of 20 (EL-20), 40, 80, and 100 U/100 g wt., chord compliance between 50 and $70 \%$ of total lung capacity (TLC) $\left(\mathrm{C}_{50-70}\right)$ and that between 80 and $100 \%$ TLC $\left(\mathrm{C}_{80-100}\right)$ were measured. After a significant initial reduction, the weight of the EL- 80 group recovered to the control level, whereas the weight curve of the EL-100 significantly decreased below that of the EL-80. Lung volumes of all the elastase-treated groups were significantly larger than those of control. Air-filled lung volumes monotonously increased when the elastase dose was increased from 20 to $80 \mathrm{U} / 100 \mathrm{~g}$ wt. In contrast lung volume of the EL-100 was significantly lower than that of the EL-80. On the other hand, liquid-filled lung volumes monotonously increased from 20 to $100 \mathrm{U} / 100 \mathrm{~g}$ wt. $\mathrm{C}_{80-100}$ was significantly smaller in the EL-100 than in the EL-80. The mean linear intercept increased and alveolar surface area decreased monotonously over the dose range tested. We conclude that there is a critical dose of elastase below which lung volume is increased and above which the increase is suppressed when elastase is administered intratracheally to Wistar rats. —— pulmonary emphysema ; mean linear intercept; lung compliance; alveolar surface area
\end{abstract}

Elastase-induced lung injury has been widely used as a disease model which resembles in many aspects human pan-acinar emphysema (Kuhn and Tavassoli 1976 ; Karlinsky and Snider 1978). However, different investigators have used widely varying amounts of porcine pancreatic elastase ranging from 1.3 to $100 \mathrm{U}$ per $100 \mathrm{~g}$ of body weight (Hayes et al. 1975; Fonzi and Lungarella 1980 ; Harkema et al. 1984 ; Kimmel et al. 1985 ; Bellofiore et al. 1989). It is known that endotracheal administration of elastase activates elastin and collagen metabolism and alters the content of these proteins to various extents (Kuhn et al. 1976 ; $\mathrm{Yu}$ and Keller 1978). Collagen is remarkably increased ( $\mathrm{Yu}$ and Keller 1978;

Received March 20, 1990; revision accepted for publication May 25, 1990. 
Valentine et al. 1983), but collagen fibers are fragmented (Bush et al. 1984). With $10 \mathrm{U}$ of elastase per $100 \mathrm{~g}$ of body weight lung elastin is decreased (Fonzi and Lungarella 1980), however, with $25 \mathrm{U}$ elastin is initially decreased and later normalized (Kuhn et al. 1976) and with $35 \mathrm{U}$ this protein is increased (Valentine et al. 1983).

Since Mead (1961) theorized that the elastic network with its high distensibility accounts for the steep portion of the lung pressure-volume curve ( $p-v$ curve) in its mid-lung volume range and the collagen with its great tensile strength accounts for the flat upper portion of the $\mathrm{p}-\mathrm{v}$ curve, several studies have demonstrated the validity of this theory. According to this theory changes in collagen and elastin should cause substantial alterations of lung mechanical properties. However, the relationship of mechanical behavior to elastase dose has not been thoroughly studied. Although the extent of the lesions produced by endotracheal elastase instillation is reported to relate morphologically to the dose of the instilled enzyme (Kaplan et al. 1973; Hayes et al. 1975), few studies have addressed this question (Damon et al. 1982 ; Raub et al. 1982). In the present study we investigated the dose-dependency of lung pressure-volume characteristics and morphometry on an elastase dose range of from 20 to $100 \mathrm{U}$ per $100 \mathrm{~g}$ of body weight in rats. In this study we confirmed the previously reported results and provide new informations on the effect of elastase on lung mechanics.

\section{Materials and Methods}

Sixty-seven male Wistar rats at postnatal week eleven were divided into one group subjected to lung pressure-volume curve studies $(n=37)$ and another group subjected to morphometric evaluation $(n=30)$. A single dose of porcine pancreatic elastase, $129.7 \mathrm{U} / \mathrm{mg}$ (United States Biochemical Corporation, Cleveland, OH, USA), was administered intratracheally to the elastase-treated groups under intraperitoneal pentobarbital anesthesia (5 mg/ $100 \mathrm{~g}$ body weight) via a tracheal catheter inserted through the mouth to a level slightly proximal to the tracheal bifurcation. During instillation, animals were held head up with their long axis oriented 60 degrees to the horizontal. Half of the elastase solution was instilled in the right lateral position and the remainder in the left lateral position. Doses ranged from 20 to $100 \mathrm{U}$ of elastase in $0.2 \mathrm{ml}$ normal saline per $100 \mathrm{~g}$ body weight. To the control group the same volume of saline was administered. Three animals were housed in a cage. The cages and bedding were changed weekly. The animals were supplied food and water ad libitum. The rats were weighed every other day. The functional and histological studies were performed four weeks after the tracheal instillation.

Lung $p-v$ curve. The method used for the measurement of the $\mathrm{p}-\mathrm{v}$ curve was as previously described (Sato et al. 1988). Briefly, intraperitoneal pentobarbital sodium anesthesia ( $5 \mathrm{mg} / 100 \mathrm{~g}$ body weight) was followed by tracheal cannulation. A suture was placed around the trachea to prevent air leakage. The animal was mechanically ventilated (Small Animal Respirator ; Harvard Instrument Co., Boston, MA, USA) with 100\% oxygen for $10 \mathrm{~min}$ at a respiratory rate of $60 / \mathrm{min}$. The tidal volume was adjusted to $25 \%$ of predicted total lung capacity (Stahl 1967). After the 5 min period of degassing by oxygen absorption with the airway occluded at end-expiration, the anterior side of the thorax was removed and the vertebral column was severed at the lumbar-thoracic junction. The preparation was discarded if the lungs did not appear small and liver-like. Immediately after degassing, $\mathrm{p}-\mathrm{v}$ curves were obtained with a gas mixture of $5 \% \mathrm{CO}_{2}, 21 \% \mathrm{O}_{2}$ and $\mathrm{N}_{2}$ 
saturated with water at room temperature in a semi-closed plastic box containing warmed water. The air inside the box was kept at $37 \pm 0.5^{\circ} \mathrm{C}$ and saturated with water vapor. The lungs were inflated at a speed of $4.76 \mathrm{ml} / \mathrm{min}$ to the transpulmonary pressure (Ptp) of 30 $\mathrm{cmH}_{2} \mathrm{O}$ then deflated to $5 \mathrm{cmH}_{2} \mathrm{O}$ Ptp and one more inflation-deflation cycle was repeated. On the third deflation from $30 \mathrm{cmH}_{2} \mathrm{O}$ the lungs were deflated to zero $\mathrm{cmH}_{2} \mathrm{O}$ Ptp by a series of $5 \mathrm{sec}$ withdrawals of $0.39 \mathrm{ml}$, each followed by $5 \mathrm{sec}$ for stress recovery. The last stepwise deflation limb was subjected to the analyses. The gas volume at a $\mathrm{Ptp}$ of $30 \mathrm{cmH}_{2} \mathrm{O}$ was defined as total lung capacity (TLC). Tracheal pressure was monitored with a differential pressure transducer (TP 603T; Nihon-Kohden Tokyo). Volume was monitored with a potentiometer (CPP-35 ; Midori Sokki Tokyo) coupled to the movement of a glass syringe mounted in the infusion-withdrawal pump. The pressure and volume signals were recorded on a pen-recorder (Recti Horiz 8S ; San-ei Sokki, Tokyo). The quasi-static chord compliances between 50 and $70 \%$ TLC $\left(\mathrm{C}_{50-70}\right)$ and between 80 and $100 \%$ TLC $\left(\mathrm{C}_{80-100}\right)$ were measured as the slope of the straight lines joining the 50 and $70 \%$ TLC points and $80-100 \%$ TLC points on the $\mathrm{p}-\mathrm{v}$ curve.

Morphometry. Intraperitoneal pentobarbital anesthesia was followed by tracheostomy, intubation of 14 gauge plastic tubing, removal of the anterior chest wall and opening of the abdomen. Subsequently the lungs were fixed intratracheally in situ at a pressure of $20 \mathrm{cmH}_{2} \mathrm{O}$ with $2.5 \%$ glutaraldehyde in $0.1 \mathrm{M}$ cacodylate buffer $\mathrm{pH} 7.4$. The speed of infusion of the fixative was high, $120 \mathrm{ml} / \mathrm{min}$ at $20 \mathrm{cmH}_{2} \mathrm{O}$ when the infusion system was not connected to the trachea (Hayatdavoudi et al. 1980). Fifteen min later the trachea was ligated, the lungs were removed from the thorax and the whole lung was submerged in the same fixative. For histological examination the lungs were trimmed of the heart and attached extraneous tissue. Total lung volume (TLV) was determined using the volume displacement method (Sherle 1970). Three $5 \mu \mathrm{m}$ sagittal sections from each left lung were cut and stained with hematoxylin and eosin. From each section sixteen pictures, resulting in forty eight pictures for each animal, were taken. To measure mean linear intercept (Lm) a fixed test line system was superimposed on the pictures and intersections of the alveolar septa were counted. Thereafter, internal surface area ( $\mathrm{Sw})$ was calculated from $\mathrm{Lm}$ and TLV.

Values are given in terms of mean \pm S.D. All the statistical analyses were performed using analysis of variance (ANOVA). A level of $p<0.05$ was considered significant.

\section{Results}

Thirty-one animals were subjected to the pressure volume analysis. Two rats of the EL-20 and one each of the EL-40 and the EL-80 groups were discarded because of gas leakage during the $\mathrm{p}-\mathrm{v}$ maneuver. Two rats of the EL-100 group died within a week after the elastase instillation. No animal treated with less than $100 \mathrm{U}$ of elastase died before sacrifice. Body weights of the five groups were similar at the time of instillation (no significant differences) (Table 1). Body weight was lost after treatment. Two days after instillation loss of body weight in the EL-80 and the EL-100 groups was significantly larger than that of the saline group $(p<0.01)$. Although the body weight of the EL-80 group recovered to the saline range by the first week, the weight of the EL-100 group did not return to the control level and the curve deviated downward from other curves. The weight recovery of the EL-100 group was significantly smaller throughout the experiment than that of the saline group $(p<0.05)$, and the recovery rate at 21 and 28 days after treatment was significantly smaller than that of the EL-80 group 
TABLE 1. Mean and S.D. of body weight and body length

\begin{tabular}{lcccc}
\hline \multirow{2}{*}{ Group } & $\begin{array}{c}\text { Number of } \\
\text { animals }\end{array}$ & $\begin{array}{c}\text { Initial } \\
(\mathrm{g})\end{array}$ & $\begin{array}{c}\text { Final wt. gain } \\
(\%)\end{array}$ & $\begin{array}{c}\text { Fody } \\
(\mathrm{mm})\end{array}$ \\
\cline { 3 - 5 } & 7 & $239 \pm 3$ & $28.2 \pm 1.7$ & $216 \pm 3$ \\
Saline & 5 & $239 \pm 14$ & $25.4 \pm 1.8$ & $217 \pm 3$ \\
EL-20 & 6 & $245 \pm 6$ & $26.5 \pm 6.6$ & $220 \pm 1$ \\
EL-40 & 6 & $244 \pm 8$ & $28.5 \pm 4.4$ & $220 \pm 2$ \\
EL-80 & 7 & $252 \pm 11$ & $20.8 \pm 3.5^{*} \dagger$ & $218 \pm 4$ \\
EL-100 & 7 &
\end{tabular}

Initial wt., wt. at elastase instillation. Final wt., wt. at measurement. ${ }^{*} p<0.05$ vs. saline, $\dagger p<0.05$ vs. EL-80. EL-20, EL-40, EL-80, and EL-100 represent groups treated with $20,40,80$, and $100 \mathrm{U}$ of elastase per $100 \mathrm{~g}$ body weight, respectively.

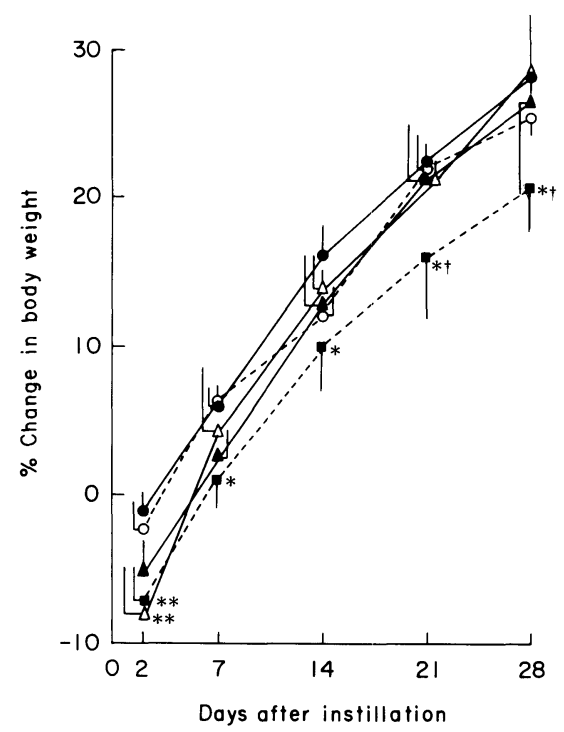

Fig. 1. Percent body weight changes after elastase instillation. Saline represents the group instilled with saline $(\bullet)$ EL-20 $(\circ)$, EL-40 (ム), EL-80 $(\triangle)$, and EL-100 (-) show the groups treated with 20, 40, 80, and $100 \mathrm{U}$ of elastase per $100 \mathrm{~g}$ body weight, respectively. Data points are means \pm s.D. ${ }^{*} p<0.05$ vs. saline $;{ }^{* *} p<0.01$ vs. saline $; \dagger p<0.05$ vs. EL-80.

(Fig. 1). Final nose-to-anus length did not significantly differ among groups (Table 1).

Lung volumes measured by air filling and corrected for body length of all the groups treated with elastase were significantly larger than those of the saline group at all Ptp tested, except at $2 \mathrm{cmH}_{2} \mathrm{O}(p<0.01$, Fig. 2 and Table 2$)$. The result was the same with correction for the body weight or without any size-corrections. 


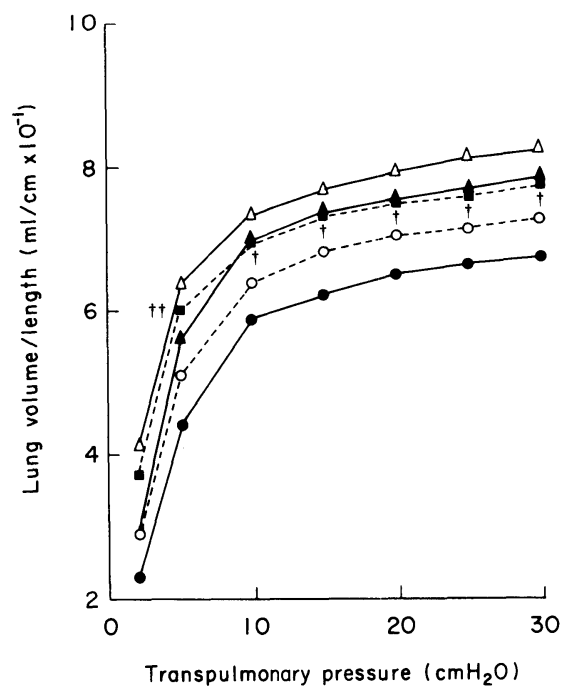

Fig. 2. Pressure-volume curve. Lung volumes are corrected for nose-to-anus length. Symbols are the same as those in Fig. $1 . \dagger p<0.05$ vs. EL-80; $\dagger+p<0.01$ vs. EL-80.

TABLE 2. Mean and S.D. of TLC and compliances

\begin{tabular}{lclcl}
\hline Group & $\begin{array}{c}\text { Number of } \\
\text { animals }\end{array}$ & \multicolumn{1}{c}{$\begin{array}{c}\mathrm{TLC} \\
(\mathrm{ml})\end{array}$} & $\begin{array}{c}\mathrm{C}_{50-70} / \mathrm{L} \\
\left(\mathrm{ml} / \mathrm{cmH}_{2} \mathrm{O} / \mathrm{m}\right)\end{array}$ & $\mathrm{C}_{80-100} / \mathrm{L}$ \\
\hline Saline & 7 & $14.6 \pm 0.2$ & $6.58 \pm 0.44$ & $0.59 \pm 0.01$ \\
EL-20 & 5 & $15.8 \pm 0.3^{* *}$ & $7.22 \pm 0.04$ & $0.62 \pm 0.01$ \\
EL-40 & 6 & $17.4 \pm 0.8^{* *}$ & $10.04 \pm 1.88^{* *}$ & $0.66 \pm 0.02^{* *}$ \\
EL-80 & 6 & $18.2 \pm 0.6^{* *}$ & $9.37 \pm 1.22^{* *}$ & $0.68 \pm 0.02^{* *}$ \\
EL-100 & 7 & $16.9 \pm 0.5^{* *} \ddagger$ & $9.50 \pm 1.46^{* *}$ & $0.62 \pm 0.02^{*} \ddagger$ \\
\hline
\end{tabular}

$\mathrm{L}$, nose to anus length. ${ }^{*} p<0.05$ and ${ }^{* *} p<0.01$ when compared with saline. $\ddagger p<0.01$ when compared with EL-80.

The corrected lung volumes of the EL-100 group were significantly smaller than those of the EL-80 group above Ptp of $5 \mathrm{cmH}_{2} \mathrm{O}(p<0.01$ at Ptp $=5$ and $p<0.05$ above $\left.10 \mathrm{cmH}_{2} \mathrm{O}\right)$. $\mathrm{C}_{50-70}$ corrected for the body length $\left(\mathrm{C}_{50-70} / \mathrm{L}\right)$ of the elastase-treated groups was significantly higher than that of the saline group except for that of the EL-20 group. However, no significant difference was observed between $\mathrm{C}_{50-70} / \mathrm{L}$ of the EL-80 and EL-100 group in contrast with the significant difference $(p<0.01)$ in $\mathrm{C}_{80-100} / \mathrm{L}$ between these two groups (Table 2 ). The increase in lung volume of the elastase-treated lungs was closely related to the rise in compliances. A significant positive correlation was found between TLC corrected for body length and $\mathrm{C}_{50-70} / \mathrm{L}(\mathrm{r}=0.72, p<0.01)$ and $\mathrm{C}_{80-100} / \mathrm{L}(\mathrm{r}=0.84$, $p<0.01)$.

The mean linear intercept tended to increase monotonously with increase of 
TABLE 3. Mean and S.D. of morphometry

\begin{tabular}{lcccc}
\hline Group & $\begin{array}{c}\text { Number of } \\
\text { animals }\end{array}$ & $\begin{array}{c}\text { Lm } \\
(\mu \mathrm{m})\end{array}$ & $\begin{array}{c}\text { TLV } \\
(\mathrm{ml})\end{array}$ & $\begin{array}{c}\mathrm{Sw} \\
\left(\mathrm{m}^{2}\right)\end{array}$ \\
\hline Saline & 6 & $72.3 \pm 3.4$ & $8.2 \pm 0.90$ & $0.45 \pm 0.06$ \\
EL-20 & 6 & $79.4 \pm 3.7$ & $9.0 \pm 0.66$ & $0.45 \pm 0.03$ \\
EL-40 & 6 & $87.3 \pm 12.2$ & $9.4 \pm 0.85^{* *}$ & $0.44 \pm 0.08$ \\
EL-80 & 6 & $97.7 \pm 17.5^{*}$ & $10.0 \pm 0.69^{* *}$ & $0.42 \pm 0.08$ \\
EL-100 & 5 & $106.6 \pm 20.4^{* *}$ & $10.4 \pm 0.34^{* *}$ & $0.40 \pm 0.09$ \\
\hline
\end{tabular}

Lm, mean liner intercept; TLV, total lung volume measured by water displacement method; Sw, alveolar surface area. ${ }^{*} p<0.05 ;{ }^{* *} p<0.01$ as compared with saline group.

elastase dose and, reversely, alveolar surface area tended to decrease monotonously. However, only $\mathrm{Lm}$ in the EL-80 and EL-100 groups was significantly larger than that of the saline group. TLV monotonously increased over the whole elastase-dose range tested in contrast to TLC. Morphologically there was no significant difference between the EL-80 and EL-100 groups (Table 3).

\section{Discussion}

The present study demonstrated that the increase in air-filled lung volume was not monotonous but phasic when Wistar rats were treated with increasing doses of elastase, from 20 to $100 \mathrm{U}$ per $100 \mathrm{~g}$ of body weight, four weeks before lung volume measurement. The turning point was between 80 and $100 \mathrm{U}$. Air-filled lung volumes above $5 \mathrm{cmH}_{2} \mathrm{O}$ of Ptp and chord compliance between 80 and $100 \%$ of TLC were significantly smaller in the EL-100 than in the EL-80 group. On the other hand, TLV, lung volume measured by water displacement after liquid filling, tended to be larger in the EL-100 group than in the EL-80 group. We are not aware of any previous reports of the dose-dependency of the compliance at the mid and at the upper most portions of the $p-v$ curve in elastase-induced emphysema. Body weight recovery also suggested the existence of a critical dose of between 80 and $100 \mathrm{U}$, i.e., animals treated with $100 \mathrm{U}$ recovered significantly less body weight than did animals treated with $80 \mathrm{U}$. Judging from the body weight curve, it appears that a physiologically greater stress was loaded to the EL-100 than to the EL-80 group. These results imply that if the severity of emphysema produced by intratracheal elastase is estimated only by the increase in lung volume we may be misled.

Lung volume measured by water displacement method were 54 to $62 \%$ of TLC. When lungs were fixed with glutaraldehyde in cacodylate buffer at 20 $\mathrm{cmH}_{2} \mathrm{O}$, the lungs inflated to $88 \%$ of TLC, gas volume measured at $25 \mathrm{cmH}_{2} \mathrm{O}$ of Ptp (Hayatdavoudi et al. 1980). We regularly observed a decrease in lung dimensions as the lungs were removed from the pressure manifold after fixation, indicating that the lungs did not retain their volume after depressurization. We 
believe that the systematically smaller TLV than TLC was due to a decrease in volume that occurred after the lungs were removed from the pressure manifold, a relatively low fixation pressure, use of glutaraldehyde and the exsanguination before fixation (Hayatdavoudi et al. 1980). Thus $\mathrm{Lm}$ in the present study represents the values at Ptp lower than approximately $7-8 \mathrm{cmH}_{2} \mathrm{O}$. For clearer estimation of the contribution of collagen fibers to the upper portion of lung $\mathrm{p}-\mathrm{v}$ curve, morphological data obtained from the lung fixed at higher fixation pressure are necessary.

Morphological findings at all elastase levels were consistent with emphysema. The values of $\mathrm{Lm}$ and Sw were similar to other reports (Harkema et al. 1984). Judging from Lm and Sw, morphological change progressed monotonously over the whole dose range tested. The tendency was that when the more elastase was given to the animals, the longer the distance between alveoli was and the smaller the total alveolar surface area was.

Our results differ from other reports which address the dependency of lung volume on elastase dose. Raub et al. (1982) reported a monotonous increase in total lung capacity and lung compliance in hamsters with increasing dose of elastase. In their study the dose of elastase ranged from 6 to $24 \mathrm{U}$ per $100 \mathrm{~g}$ weight, which is much lower dose than that used in the present study. Differences in elastase dose range and animal species may account for the discrepancy. On the other hand a careful examination of the data shown by Damon et al. (1982) seems to suggest the possibility that the relationship between the increase in lung volume and elastase dose might be phasic with increasing elastase dose, from 30 to $120 \mathrm{U}$ per $100 \mathrm{~g}$ body weight. Lung volume increased monotonously with dosages of from 30 to $100 \mathrm{U}$ elastase, but slightly decreased when the elastase dose was raised to $120 \mathrm{U}$, although the number of animal tested was only two. They stated that the lung volumes generally increased with increasing elastase dose.

We will next discuss the reasons why TLC and $\mathrm{C}_{80-100}$, which were the parameters obtained at the upper portion of $\mathrm{p}-\mathrm{v}$ curve was smaller in the EL-100 group. As in the present study, an increase in lung volume has been reported in living animals treated with intratracheal elastase (Kaplan et al. 1973; Hayes et al. 1975; Snider and Sherter 1977; Harkema 1984; Yokoyama et al. 1987). However, when elastase was instilled intratracheally to excised lungs no increase in TLC was observed. Only an increase in the chord compliance at the mid portion of the $\mathrm{p}-\mathrm{v}$ curve was observed (Karlinsky et al. 1976). The disparity between the differences in TLC measured in an in vitro system and that measured in living animals was explained on the basis of a modification of collagen metabolism (Karlinsky et al. 1976). Although the present study does not provide data directly clarifying the cause of this discrepancy, one of the mechanisms by which $\mathrm{C}_{80-100}$ in the EL-100 group decreased to below that in the EL-80 group seems to be related to this point. The compliance in this portion of the $\mathrm{p}-\mathrm{v}$ curve 
has been considered to be determined mainly by collagen (Mead 1961 ; Martin and Sugihara 1973; Karlinsky et al. 1976).

Intratracheal instillation of elastase activates the collagen metabolism and leads to an increase of collagen in the lung (Kuhn et al. 1976; Yu and Keller 1978; Fonzi and Lungarella 1980). In order to explain the monotonously increasing TLC with up to $80 \mathrm{U}$ of elastase as observed in the present study, together with the reported increase in biochemically measured collagen, it is necessary to take into account damage to collagen fibers by collagenase elaborated during acute cellular response (Robertson et al. 1972 ; Senior et al. 1972 ; Wahl et al. 1975). We speculate that the damage of collagen fibers by collagenase was enhanced monotonously with increase in elastase dose from 20 to $80 \mathrm{U}$, but inhibited with further increase of elastase, resulting in the low compliance at high Ptp range. Possibly the cellular response responsible for collagenase activation is modified. Remodeling of the lung structure during repairment from injury may also be responsible for the lowered compliance at high Ptp with elastase dosage of $100 \mathrm{U}$ (Karlinsky 1976).

Finally we will discuss the reasons why the air-filled lung volumes of the EL-100 group was smaller than that of the EL-80 group even at the mid or lower portion of p-v curve. TLV of the EL-100 group was not smaller than TLV of the EL-80 group. This was a conspicuous contrast to TLC. TLC is a lung volume measured by air filling, whereas TLV is a lung volume measured by liquid filling. When lungs are inflated by liquid, most of the elastic recoil pressure arising from lung surfactant is removed and the recoil pressure at a given lung volume reflects mainly the tissue recoil pressure at that lung volume. Although in the present study lungs were not completely degassed before fixation but we started the fixation with the minimum volume of the lungs which had been exsanguinated, most of the air-liquid interface should have been eliminated. Then, in order to explain the smaller air-filled lung volume in the EL-100 than that in the EL-80 group range, it is necessary to take into account differences in the contribution of surfactant to the air-filled $\mathrm{p}-\mathrm{v}$ curve. In vitro surface tension coefficient $(\gamma)$ surface area (A) curve of the lung surfactant is shifted toward higher minimum surface tension coefficient by several factors, such as the amount of active material present or the action of blocking substances (Klaus et al. 1961). If this kind of leftward shift of $\gamma$-A curve occurred in the EL-100 group, then the air-filled lung $\mathrm{p}-\mathrm{v}$ curve would shift toward higher Ptp range. In other words, lung volume at a given Ptp would be estimated smaller as shown in the EL-100 group. It is quite possible that in the lung damaged by elastase the amount and the quality of surfactant are altered by extensive stretching of the alveolar wall to which alveolar Type II cells are attached and remodeling of the alveolar space (Snider and Korthy 1978). However, little has been investigated on the surfactant properties of the animals treated with intratracheally instilled elastase. Studies addressing the dose-dependency of the damages given by elastase to the alveolar 
Type II cells and the potency of the surfactant produced in the remarkably enlarged air spaces are necessary to elucidate this problem.

\section{References}

1) Bellofiore, S., Eidelman, D.H., Macklem, P.T. \& Martin, J.G. (1989) Effects of elastase-induced emphysema on airway responsiveness to methacholine in rats. $J$. Appl. Physiol., 66, 606-612.

2) Bush, R.H., Lauhala, K.E., Loscutoff, S.M. \& McDonald, K.E. (1984) Experimental pulmonary emphysema induced in the rat by intratracheally administered elastase : Morphogenesis. Environ. Res., 33, 497-513.

3) Damon, E.G., Mauderly, J.L. \& Jones, R.K. (1982) Early effects of intratracheal instillation of elastase on mortality, respiratory function, and pulmonary morphometry of F-344 rats. Toxicol. Appl. Pharmacol., 64, 465-475.

4) Fonzi, L. \& Lungarella, G. (1980) Correlation between biochemical and morphological repair in rabbit lungs after elastase injury. Lung, 158, 165-171.

5) Harkema, J.R., Mauderly, J.L., Gregory, R.E. \& Pickrell, J.A. (1984) A comparison of starvation and elastase models of emphysema in the rat. Am. Rev. Respir. Dis., 129, 584-591.

6) Hayatdavoudi, G., Crapo, J.D., Miller, F.J. \& O'Neil, J. (1980) Factors determining degree of inflation in intratracheally fixed rat lungs. J. Appl. Physiol.: Respirat. Environ. Exercise Physiol., 48, 389-393.

7) Hayes, J.A. Korthy, A. \& Snider, G.L. (1975) The pathology of elastase-induced panacinar emphysema in hamsters. J. Pathol., 117, 1-14.

8) Kaplan, P.D., Kuhn, C. \& Pierce, J.A. (1973) The induction of emphysema with elastase. 1. The evolution of the lesion and the influence of serum. J. Lab. Clin. Med., 82, 349-356.

9) Karlinsky, J.B., Snider, G.L., Franzblau, C., Stone, P.J. \& Hoppin F.G., Jr. (1976) In vitro effects of elastase and collagenase on mechanical properties of hamster lungs. Am. Rev. Respir. Dis., 113, 769-777.

10) Karlinsky, J.B. \& Snider, G.L. (1978) Animal models of emphysema. Am. Rev. Respir. Dis., 117, 1109-1133.

11) Kimmel, E.C., Winsett, D.W. \& Diamond, L. (1985) Augmentation of elastaseinduced emphysema by cigarette smoke. Am. Rev. Respir. Dis., 132, 885-893.

12) Klaus, M.H., Clements, J.A. \& Havel, R.J. (1961) Composition of surface active material isolated from beef lung. Proc. Natl. Acad. Sci. USA, 47, 1858-1859.

13) Kuhn, C., III \& Tavassoli, F. (1976) The scanning electron microscopy of elastaseinduced emphysema. Lab. Invest., 34, 2-9.

14) Kuhn, C., III, Yu, Shiu-Yeh, Chraplyvy, M., Linder, H.E. \& Senior, R.M. (1976) The induction of emphysema with elastase. II. Changes in connective tissue. Lab. Invest., 34, 372-380.

15) Martin, C.J. \& Sugihara, T. (1973) Simulation of tissue properties in irreversible diffuse obstructive pulmonary syndromes: Enzyme digestion. J. Clin. Invest., 52, 1918-1924.

16) Mead, J. (1961) Mechanical properties in lungs. Physiol. Rev., 41, 281-330.

17) Raub, J.A., Mercer, R.R., Miller, F.J., Graham, J.A. \& O'Neil, J.J. (1982) Dose response of elastase-induced emphysema in hamsters. Am. Rev. Respir. Dis., 125, $432-435$.

18) Robertson, P.B., Ryel, R.B., Taylor, R.E., Shyu, K.W. \& Fullmer, H.M. (1972) Collagenase: Localization in polymorphonuclear leucocyte granules in the rabbit. Science, 177, 64-65.

19) Sato, S., Nakamura, H., Takada, K. \& Takahashi, K. (1988) Dietary vitamin E 
supplement does not inhibit changes in lung pressure-volume characteristics produced by bleomycin in hamsters. Eur. Respir. Dis., 1, 523-526.

20) Senior, R.M., Bielefeld, D.R. \& Jeffrey, J.J. (1972) Collagenolytic activity in alveolar machrophages. Clin. Res., 20, 88.

21) Sherle, W.A. (1970) A simple method of volumetry of organs in quantitative stereology. Makroskopie, 26, 57-60.

22) Snider, G.L. \& Sherter, C.B. (1977) A one-year study of the evolution of elastaseinduced emphysema in hamsters. J. Appl. Physiol.: Respirat. Environ. Exercise Physiol., 43, 721-729.

23) Snider, G.L. \& Korthy, A.L. (1978) Internal surface area and number of respiratory air spaces in elastase-induced emphysema in hamsters. Am. Rev. Respir. Dis., 117, 685-693.

24) Stahl, W.R. (1967) Scaling of respiratory variables in mammals. J. Appl. Physiol., 22, 453-460.

25) Valentine, R., Rucker, R.B., Chrisp, C. E. \& Fisher, G.L. (1983) Morphological and biochemical features of elastase induced emphysema in strain A/J mice. Toxic. Appl. Pharmacol., 68, 451-461.

26) Wahl, L.M., Wahl, S.M., Mergenhagen, S.E. \& Martin, G.R. (1975) Collagenase production by lymphokine-activated machrophages. Science, 187, 261-263.

27) Yokoyama, E., Nambu, Z., Uchiyama, I. \& Kyono, H. (1987) An emphysema model in rats treated intratracheally with elastase. Environ. Res., 42, 340-352.

28) $\mathrm{Yu}$, Shiu-Yeh \& Keller, N.R. (1978) Synthesis of lung collagen in hamsters with elastase-induced emphysema. Exp. Mol. Pathol., 29, 37-43. 